\title{
Continuations intra et interphrastiques du français : premiers résultats expérimentaux
}

\author{
Dargnat, Mathilde ${ }^{1}$, Colotte, Vincent ${ }^{2}$, Bartkova, Katarina ${ }^{1} \&$ Bonneau, Anne $^{3}$ \\ 1. Université de Lorraine \& ATILF CNRS, 2. Université de Lorraine \& LORIA CNRS, 3. LORIA CNRS \\ \{mathilde.dargnat;katarina.bartkova\}@atilf.fr, \{vincent.colotte;anne.bonneau\}@loria.fr
}

\section{Introduction}

\subsection{Problématique de départ}

Une étude préalable (Dargnat 2008, Dargnat \& Jayez 2010, Jayez \& Dargnat à par. 2012) basée sur des données de français oral spontané a permis de mettre en évidence un patron prosodique récurrent à la fin du premier élément de ce qui est traditionnellement appelé une juxtaposition, à savoir « une phrase complexe formée d'une suite de deux ou plusieurs propositions qui pourraient être considérées chacune comme une phrase autonome [...] mais dont le rapport n'est pas explicitement marqué par un mot de relation » (Riegel et al. 2009 : 781). En effet, dans des constructions de type XY ou X et Y sont deux phrases déclaratives reliées par une relation de discours non marquée lexicalement $(1,2,3,4)$, la fin de la phrase X présente le plus souvent un patron montant et il n'y a pas de pause perceptible entre X et Y.

[j'en ai pas $]_{\mathrm{X}}\left[\mathrm{j}^{\prime} \text { en ai pas les moyens }\right]_{\mathrm{Y}}$ (corpus Strip Tease, stI3-9'44)

[j'ai un coup de cafard $]_{\mathrm{X}}$ [je mets une cassette vidéo jusque les trois quatre heures du matin $]_{\mathrm{Y}}$ (corpus Strip Tease, stI3-33'54)

[je m'occupais des personnes âgées $]_{\mathrm{X}}$ [on les levait le matin $]_{\mathrm{Y}}$ (Corpus Allier (Giron 2004), A$\operatorname{mad}-2 / 6-1,08)$

(4) [Flore avait commandé des Petshops $]_{\mathrm{X}}$ [elle a eu des Playmobils $]_{\mathrm{Y}}$ (entendu Noël 2009)

Des analyses plus spécifiques proposent parfois d'autres termes pour étiqueter ces constructions et en différencier éventuellement plusieurs sous-catégories. Généralement, on parle de parataxes pour insister sur le caractère autonome de $\mathrm{X}$ et $\mathrm{Y}$ et/ou d'asyndètes pour mettre l'accent sur l'absence de mot de liaison (pour une histoire des termes et une synthèse des propriétés, voir Glikman 2009, Benzitoun 2010). Dans les approches macro-syntaxiques, on trouve également les termes de couplage, structures binaires et greffes qui s'appliquent plus ou moins à des cas similaires (Deulofeu 1977, 1989, 1999, Avanzi 2011).

Dans tous les cas, le phénomène est présenté comme étant à l'interface entre syntaxe, discours et prosodie. Les deux angles d'attaque les plus importants sont, d'une part, le type de dépendance entre X et Y et, d'autre part, le rôle de la prosodie dans le marquage de cette dépendance. S'agit-il d'une dépendance de type syntaxique par complémentation ou adjonction ? S'agit-il plutôt ou aussi d'une dépendance discursive ? Le patron récurrent observé est-il systématiquement associé à un sens ? Correspond-il par ailleurs à un patron déjà répertorié du français?

Il n'est pas prévu de traiter ici de la question de la dépendance syntaxique. Le patron prosodique montant est appréhendé a priori comme un contour, c'est-à-dire, pour nous, comme l'association d'une forme prosodique et d'un sens ou plus exactement d'un programme discursif. Dans de telles constructions, la montée mélodique sur la fin de X est associée à une incomplétude discursive, c'est-àdire que X est présenté comme un segment discursif non terminé (cf. Beyssade et al. 2006, DelaisRoussarie 2005 : 99-121, Marandin 2006, Portes et al. 2007). Cette interprétation est aussi celle qui soustend la notion de continuation et plus précisément la définition des/du contour(s) de continuation, et ce dès les premières définitions (voir en particulier Chen 2007, Choi-Jonin et Delais-Roussarie 2006, DelaisRoussarie 2011, Delattre 1961, 1966, Di Cristo 1998, 2010 : 5-23, Rossi 1999). 
Cet article est une contribution à l'analyse des structures non conclusives en français ${ }^{\mathrm{i}}$ sur la base d'un protocole expérimental permettant de contraster les réalisations prosodiques de plusieurs configurations traditionnellement distinguées, à savoir les configurations de continuation mineure, de continuation majeure, de question et de finalité (ou déclarative conclusive). Il ne s'agit pas de forcer une précatégorisation des phénomènes que nous étudions en fonction de la littérature existante, mais il s'agit de voir si les configurations syntaxiques et discursives que l'on peut associer aux contours proposés par Delattre, entre autres, amènent systématiquement les sujets que nous avons sollicités à produire ces contours.

\subsection{Objet de l'étude}

Le champ d'analyse est pour le moment restreint du point de vue des objets linguistiques analysés et du point de vue des propriétés prosodiques. Ces restrictions permettent néanmoins de tirer certaines conclusions, de soulever de nouvelles questions et d'envisager d'autres analyses, d'autres contrastes (voir section 5 pour les perspectives).

Les segments analysés sont de deux types, ou plus exactement opèrent à deux niveaux. Au niveau syntaxique, nous avons examiné la configuration prosodique finale des SN sujets simples dans des phrases déclaratives, suivies (5) ou non (6) d'une autre phrase déclarative. Au niveau discursif, nous avons examiné la configuration prosodique finale de la première proposition dans des phrases complexes mettant en jeu deux propositions comportant chacune un verbe conjugué, reliées entre elles par une conjonction (7) ou simplement juxtaposées (8).

Concernant les propriétés prosodiques, nous nous sommes concentrés sur l'étude de la pente des deux dernières syllabes des segments-cibles (en gras).

Chaque exemple renvoie entre crochets à un type de configuration en lien avec la problématique.

(5) Les agneaux ont vu leur mère, elle sort du bosquet. [configuration CIA]

(6) Les agneaux ont vu leur mère. [configuration CIS]

(7) Il a vu Maria, car elle vient de rentrer. [configuration CAO]

(8) Il a vu Maria, elle vient de rentrer. [configuration CAP]

Des cas plus complexes ont été construits pour pouvoir effectuer, éventuellement, des contrastes plus fins. Dans sa globalité, l'analyse repose non sur quatre mais sur huit configurations différentes qui peuvent parfois être envisagées par paires, elles sont explicitées à la section 2.1.

Nous abordons ici deux types de questions.

Premièrement, nous nous demandons si les patrons finaux ciblés possèdent les mêmes propriétés que les contours continuatifs, tels qu'ils sont présentés par Delattre $(1961,1966)$ et Rossi $(1980,1999)$ qui distinguent la continuation mineure, ou simple, et la continuation majeure, ou groupante ${ }^{\text {ii }}$. La distinction est faite en particulier au niveau de leurs formes et de leur hiérarchisation. Rappelons quelques unes des propriétés le plus souvent avancées pour les opposer : le contour de continuation mineure peut être descendant ou montant, mais il est majoritairement montant; il monte moins haut que le contour de continuation majeure ${ }^{\mathrm{iii}}$ et intervient à la fin de «groupes à sens partiel»; le contour de continuation majeure est toujours ${ }^{\text {iv }}$ montant et il intervient à la fin d'un segment de discours formant « un groupe de sens plus complet», comme une proposition. Le contour de continuation majeur peut donc être précédé d'un ou plusieurs contours de continuation mineure.

Rapporté à notre objet d'étude, cela implique que l'on fait l'hypothèse, au moins dans un premier temps, que les contours observés sur les deux dernières syllabes des SN sujets simples (configuration CIS et configuration CIA) sont apparentés à des continuations mineures et que les contours observés sur les deux dernières syllabes de la première proposition dans une phrase complexe sont apparentés à des continuations majeures (configuration $\mathrm{CAO}$ et configuration $\mathrm{CAP}$ ), dans la mesure où les segments en jeu ici sont des segments formant un groupe de sens «plus complet» que dans le cas des SN sujets. Cela 
correspond bien aux traitements de certains exemples dans la littérature sur le sujet. Une telle hypothèse amène plusieurs questions auxquelles nous répondrons sur la base des observations en production.

- Les pentes observées dans les configurations CIS-CIA peuvent-elles être montantes et descendantes ? Les hauteurs maximales de ces pentes sont-elles systématiquement inférieures aux hauteurs maximales des pentes dans les configurations CAO-CAP ?

- Les pentes observées dans les configurations CAO-CAP sont-elles toujours montantes, comme c'est le cas pour les contours de continuation majeure de Delattre ?

- Les pentes observées dans les configurations CIS-CIA et les configurations CAO-CAP sont-elles significativement différentes des pentes observées dans les configurations de question correspondantes (resp. configuration QIS et configuration QAS) et conclusives correspondantes (resp. configuration DIS et configuration DAS) ? Pour les illustrations des configurations QIS, QAS, DIS et DAS, voir ci-dessous section 2.1.

Deuxièmement, nous nous demandons si, dans le cas des phrases complexes, le fait que la relation de discours entre les deux propositions soit marquée, ici par une conjonction de coordination à sens causal, influence le patron prosodique final de la première proposition. Plus concrètement, nous nous sommes demandé si, dans le corpus recueilli, il y avait une différence systématique entre les configurations $\mathrm{CAO}$ et les configurations CAP.

Cette étude doit donc apporter des éléments sur la catégorisation du patron prosodique observé à la fin de $\mathrm{X}$ dans des structures juxtaposées de type $\mathrm{XY}$, et permettre une évaluation des catégories préexistantes que sont la continuation mineure et la continuation majeure. Elle doit aussi permettre de poser plus franchement le problème d'une éventuelle spécificité prosodique ou non des constructions asyndétiques par rapport à leur équivalent avec conjonction.

\section{Protocole expérimental}

Une étude sur corpus oral spontané peut sembler préférable pour des raisons d'authenticité des données, mais à cause de l'unicité de chaque occurrence de phénomène, il est impossible de s'en contenter lorsque l'on souhaite faire des contrastes systématiques et statistiquement exploitables en maîtrisant le mieux possible l'aspect quantitatif et les facteurs de variation de type phonétique et contextuel (cf. Marandin 2010 pour une synthèse sur ce point). C'est essentiellement pour ces raisons que nous avons pensé un protocole expérimental permettant de recueillir des données calibrées pour l'étude (choix des phrases, des segments-cibles, voire des phonèmes, etc.) et répétables par plusieurs sujets dans des conditions similaires.

\subsection{Construction de l'expérience}

En fonction des types de contrastes souhaités, nous avons défini huit configurations mettant en jeu, a priori, des cas de continuations mineures et majeures dans les positions qui nous intéressaient (SN sujet simple et fin de première proposition dans des phrases complexes), ainsi que les cas correspondants de déclaratives conclusives et de questions. Il faut rappeler que cette correspondance entre nos configurations et les continuations, comme nous l'avons dit plus haut, est une hypothèse que nous cherchons à tester et qui étend significativement les remarques de Delattre. Pour effectuer des contrastes fins, ou nous laisser la possibilité de le faire ultérieurement, nous avons aussi distingué des souscatégories en fonction de l'environnement linguistique du segment étudié. Les configurations peuvent donc être regroupées dans certaines analyses (exemples de contrastes : configurations CIA-CIS vs configurations CAO-CAP mais aussi configuration CAO vs configuration CAP).

Concrètement, nous avons formulé cinq phrases différentes par configuration, sur le même modèle. Le tableau ci-dessous n'expose que trois cas mais permet d'illustrer la fabrication des phrases enregistrées par les sujets en fonction de la configuration recherchée. Les phrases juxtaposées ont été présentées aux sujets sans ponctuation intermédiaire (pas de virgule entre les deux propositions), afin de 
ne pas les influencer relativement à la production ou non d'une pause. La ponctuation finale (point ou point d'interrogation) a été conservée pour forcer la complétude de l'unité discursive en jeu.

\begin{tabular}{|c|c|c|}
\hline Nom & Type de configuration visé & $\begin{array}{l}\text { Exemples de phrases à enregistrer } \\
\text { En tout : } 5 \text { phrases différentes par configuration }\end{array}$ \\
\hline CIS & $\begin{array}{l}\text { configuration continuative sur SN sujet simple } \\
\text { dans une phrase simple }\end{array}$ & $\begin{array}{l}\text { 1. Les agneaux ont vu leur mère. } \\
\text { 2. Nos amis aiment bien Nancy. } \\
\text { 3. Les élèves vont lire Malraux. } \\
\ldots\end{array}$ \\
\hline CIA & $\begin{array}{l}\text { configuration continuative sur SN sujet simple } \\
\text { dans la première proposition d'une phrase } \\
\text { complexe }\end{array}$ & $\begin{array}{l}\text { 1. Les agneaux ont vu leur mère elle sort } \\
\text { du bosquet. } \\
\text { 2. Nos amis aiment bien Nancy ils y ont } \\
\text { grandi. } \\
\text { 3. Les élèves vont lire Malraux c'est au } \\
\text { programme du bac. } \\
\text {... }\end{array}$ \\
\hline DIS & $\begin{array}{l}\text { configuration déclarative conclusive sur un SN } \\
\text { simple }\end{array}$ & $\begin{array}{ll}\text { 1. Les agneaux. } \\
\text { 2. Nos amis. } \\
\text { 3. Les élèves. } \\
\text {... }\end{array}$ \\
\hline QIS & configuration de question sur SN simple & $\begin{array}{ll}\text { 1. } & \text { Les agneaux ? } \\
\text { 2. } & \text { Nos amis ? } \\
\text { 3. } & \text { Les élèves? }\end{array}$ \\
\hline CAO & $\begin{array}{l}\text { configuration continuative à la fin de la première } \\
\text { proposition d'une phrase complexe avec mot } \\
\text { coordonnant }\end{array}$ & $\begin{array}{l}\text { 1. Il a vu Maria car elle est rentrée. } \\
\text { 2. Il dort chez Maria car il finit tard. } \\
\text { 3. Il relit Maria car elle fait trop de fautes }\end{array}$ \\
\hline CAP & $\begin{array}{l}\text { configuration continuative à la fin de la première } \\
\text { proposition d'une phrase complexe sans mot } \\
\text { coordonnant }\end{array}$ & $\begin{array}{l}\text { 1. Il a vu Maria elle vient de rentrer. } \\
\text { 2. Il dort chez Maria il va finir tard. } \\
\text { 3. Il relit Maria elle fait trop de fautes }\end{array}$ \\
\hline DAS & $\begin{array}{l}\text { configuration déclarative conclusive à la fin } \\
\text { d'une phrase simple }\end{array}$ & $\begin{array}{l}\text { 1. Il a vu Maria. } \\
\text { 2. Il dort chez Maria. } \\
\text { 3. Il relit Maria. } \\
\ldots\end{array}$ \\
\hline QAS & $\begin{array}{l}\text { configuration de question à la fin d'une phrase } \\
\text { simple }\end{array}$ & $\begin{array}{ll} & \text { 1. Il a vu Maria ? } \\
\text { 2. Il dort chez Maria ? } \\
\text { 3. Il relit Maria ? }\end{array}$ \\
\hline
\end{tabular}

Tableau 1. Types de configurations et illustrations

Une fois l'ensemble des phrases obtenu, nous avons créé plusieurs listes de passation, sur le même modèle, afin de croiser les sujets et éviter un effet de répétition trop important. Aucun sujet n'a prononcé deux fois la même phrase dans des configurations différentes. De plus, nous avons inséré trois phrases de test en début d'expérience, ainsi qu'un certain nombre de distracteurs (non présentés ici) pour dissimuler 
l'objectif de l'expérience. Enfin, chaque phrase a été insérée dans un contexte situationnel et dialogal pour contraindre le plus possible sa modalité et sa structure informationnelle.

Dans cet article, nous ne traitons que les données pour le français, qui ont été recueillies à Nancy en mai 2010. Dans la mesure où tous les sujets francophones natifs avaient l'anglais comme L2, la même procédure a été appliquée pour l'anglais (traduction des phrases et mise en place dans les mêmes conditions) afin de recueillir des données sur la L2. Pour des raisons relatives aux langues elles-mêmes, il a tout de même fallu adapter certaines phrases, en particulier les interrogatives sans inversion, assez naturelles en français mais «marquées » en anglais. De plus, pour compléter les données et pour nous permettre d'avoir une prononciation native de l'anglais, le même corpus de phrases en anglais a été enregistré en septembre 2011 auprès d'une vingtaine de locuteurs de l'anglais originaires de Newcastle.

Nous renvoyons à la dernière partie de la section 5 pour quelques commentaires concernant la comparaison L1/L2.

\subsection{Passation de l'expérience}

Les trente deux sujets qui ont participé volontairement sont âgés de 19 à 49 ans, sont tous des locuteurs natifs du français, et la majorité sont des étudiants de Lettres et Sciences humaines. Notons une disproportion importante dans la distribution des sexes (plus de filles de que garçons), qui tient en grande partie à la filière universitaire d'origine.

L'interface d'enregistrement a été réalisée avec le logiciel CorpusRecorder modifié pour l'occasion (développé au Loria, mais non diffusé). L'intérêt est d'abord pratique : il permet un calibrage de la voix avant l'expérience et alerte le sujet en cas de mauvais signal lors de l'enregistrement (saturation ou son trop faible). D'autre part, ce logiciel permet d'associer directement à chaque sujet un profil documentaire (informations personnelles, enregistrements, liste de phrases concernée), très utile pour le classement et le tri de l'ensemble des données recueillies. Voici une des fenêtres d'affichage demandant d'enregistrer une phrase ayant la configuration CAO.

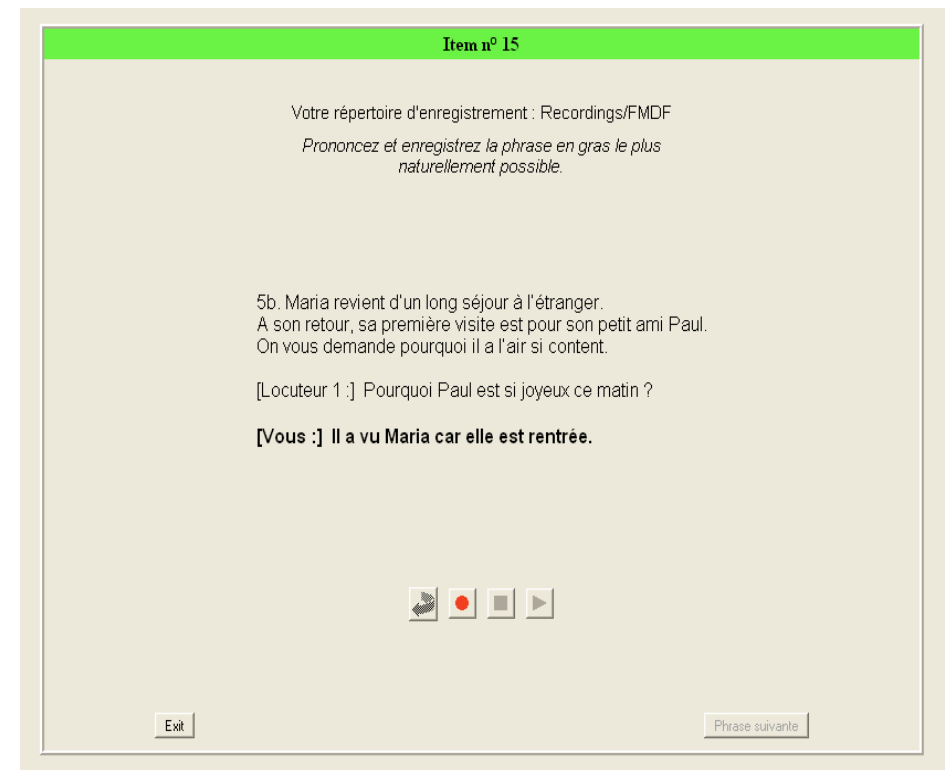

Figure 1. CorpusRecorder : interface pour l'enregistrement du corpus.

Les enregistrements ont eu lieu au sein d'un des deux laboratoires de recherche concernés par le projet (ATILF), en parallèle dans deux pièces isolées avec du matériel identique. Après quelques explications, les sujets étaient laissés seuls et avaient pour consigne de n'enregistrer que la phrase en gras, après une lecture soigneuse et une bonne compréhension du contexte. Chacun avait la possibilité de se réécouter et 
de recommencer l'enregistrement jusqu'à ce qu'il trouve sa prononciation suffisamment naturelle. Chaque sujet se voyait proposer une cinquantaine d'écrans (distracteurs compris) en deux parties : d'abord les phrases en français, puis celles en anglais. La durée moyenne de passation a été d'une demiheure. Nous avons ainsi recueilli plus de trois cents fichiers sons au format wav exploitables pour le français (et autant pour l'anglais).

\subsection{Traitement du signal}

Chaque fichier a ensuite été segmenté et annoté manuellement en phonèmes en utilisant l'API, avec le logiciel Winsnoori. La présentation finale est aussi visible sous Praat avec un codage SAMPA des phonèmes, comme le montre l'illustration ci-dessous.
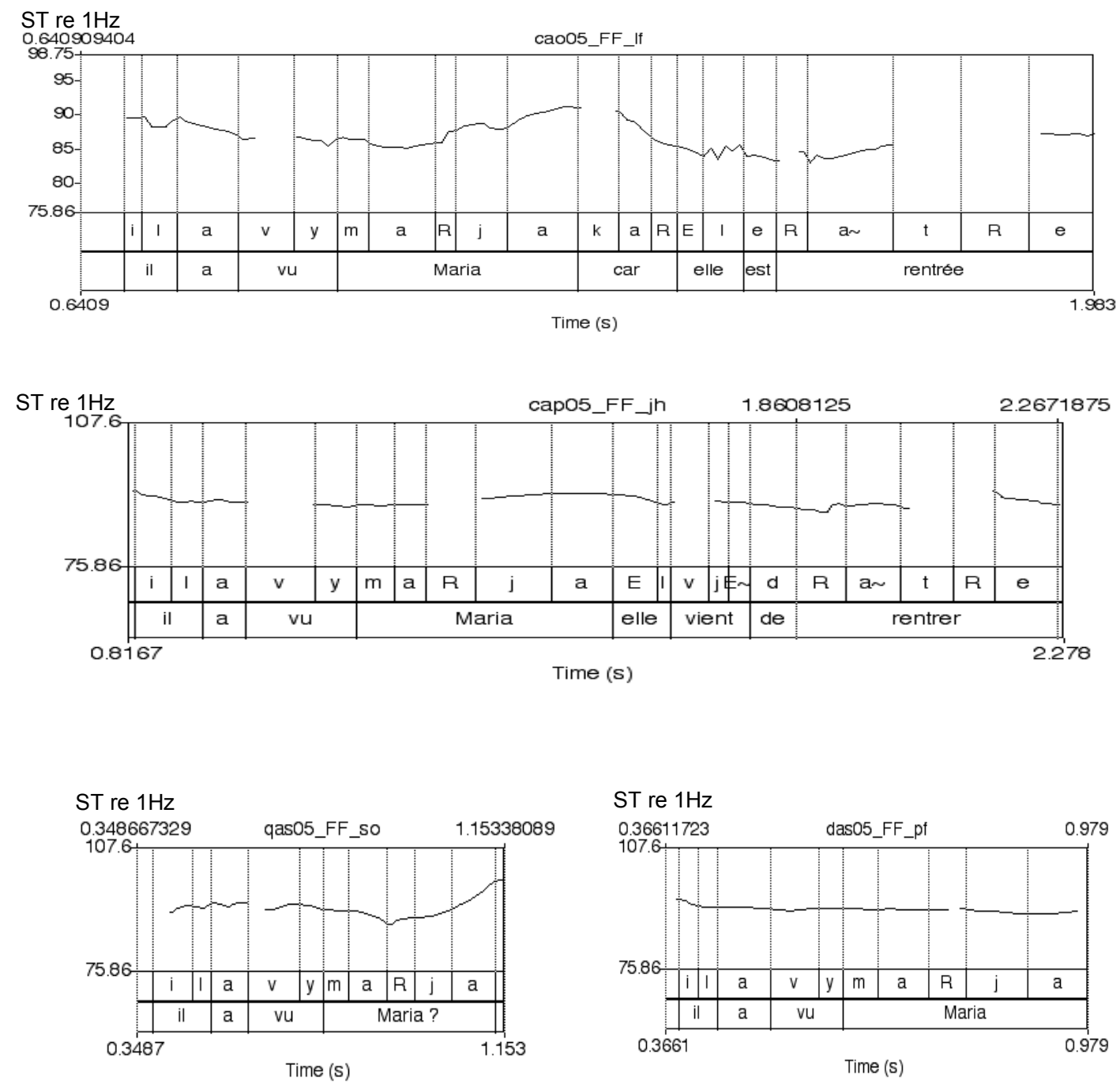

Figures 2. Segmentation, annotation et visualisation du signal avec Praat et SAMPA. 


\section{Analyse des résultats}

Comme cela est précisé dans l'introduction, nous nous sommes concentrés dans un premier temps sur les dernières syllabes de certains segments discursifs et sur la pente observée sur ces segments.

\subsection{Nature des données}

Grâce à la segmentation et à l'annotation des fichiers, nous avons récupéré automatiquement les données numériques qui nous intéressaient. Un script permet de relever la valeur de la fréquence fondamentale toutes les $10 \mathrm{~ms}$ et de calculer la droite de régression (Rslope) pour les syllabes ciblées pour chaque enregistrement. Les valeurs sont exprimées en semi-tons (ST) essentiellement pour contourner l'écart de registre entre les sujets masculins et féminins. Les données manipulées dans les tests statistiques tels qu'ils sont présentés ci-dessous sont donc les valeurs de RslopeST pour les segments-cibles de chaque occurrence de phrase enregistrée.

\subsection{Traitement statistique des contrastes}

La masse de données récupérée a été analysée avec un modèle linéaire mixte paramétré sous $R^{\mathrm{v}}$. On a pu ainsi contraster ${ }^{\mathrm{vi}}$ les différents types de configuration et montrer quelles différences étaient ou non significatives d'un point de vue statistique.

Les sorties brutes sont présentées ci-dessous sous la forme de trois figures correspondant à trois types de contrastes :

La figure 3 concerne les contrastes entre les configurations apparentées à un contour de continuation mineure (configuration CIA, CIS) et les configurations apparentées à un contour de continuation majeure (configuration $\mathrm{CAO}, \mathrm{CAP}$ ). La division en deux sous-catégories permet de faire des contrastes fins.

La figure 4 concerne les contrastes entre les configurations apparentées à un contour de continuation majeure (configuration $\mathrm{CAO}, \mathrm{CAP}$ ) et les configurations déclaratives conclusives (configuration DAS) et de question (configuration QAS) correspondantes. Ce contraste a pour objectif de mettre en évidence : (i) la distribution des contours montants et descendants parmi les configurations apparentées à des contours de continuation majeure (dans la littérature qui nous sert de référence, la continuation majeure est présentée comme devant être montante) ; (ii) une ressemblance ou non entre les pentes montantes observées pour les configurations apparentées à un contour de configuration majeure et les pentes des questions (dans la littérature qui nous sert de référence, le contour de question a la possibilité de monter plus haut que les contours de continuation) ; (iii) une ressemblance ou non entre les pentes descendantes éventuellement observées pour les configurations apparentées à un contour de configuration majeure et les pentes des déclaratives conclusives, qui, normalement, ont la propriété d'être descendantes.

La figure 5 concerne les contrastes entre les configurations apparentées à un contour de continuation mineure (configuration CIS,CIA) et les configurations déclaratives conclusives (configuration DIS) et de question (configuration QIS) correspondantes. Ce contraste a pour objectif de mettre en évidence : (i) la distribution des contours montants et descendants parmi les configurations apparentées à des contours de continuation mineure (dans la littérature qui nous sert de référence, la continuation mineure peut effectivement descendre ou monter, mais monte dans la majorité des cas); (ii) une ressemblance ou non entre les pentes montantes observées pour les configurations apparentées à un contour de configuration mineure et les pentes des questions, (iii) une ressemblance ou non entre les pentes descendantes observées pour les configurations apparentées à un contour de configuration mineure et les pentes conclusives.

Les graphiques montrent la distribution de toutes les observations en fonction de leur appartenance à un type de configuration, les tableaux donnent les résultats pour les contrastes des configurations deux à deux. 


\section{Légende}

$*$ : la différence observée est significative. Seuils : $* * *<1 \% 0_{0}<* *<1 \%<*<5 \%$

RslopeST : pente de la droite de régression calculée à partir des hauteurs en semi-tons sur le segment-cible

Ordonnées : valeurs de RslopeST ; abscisses : rangement des observations par ordre croissant (chaque point correspond à une observation)

\section{Modalités de lecture des tableaux}

Quand le résultat de la colonne de droite $(\operatorname{Pr}(>|z|)$ est suivi d'un ou plusieurs astérisques, cela veut dire que le résultat du contraste testé est inférieur à un certain seuil de significativité (indiqué dans la légende, ci-dessus). Plus Pr est faible, plus l'hypothèse qu'il n'y ait pas de différence (par ex. cap-cao $=0$ ) entre les deux configurations contrastées a des chances de ne pas être vérifiée. Donc, plus Pr est faible, plus les configurations contrastées peuvent être considérées comme différentes (du point de vue de la pente de régression en semi-tons calculée sur les deux dernières syllabes), autrement dit plus la différence observée peut être considérée comme n'étant pas due au hasard.

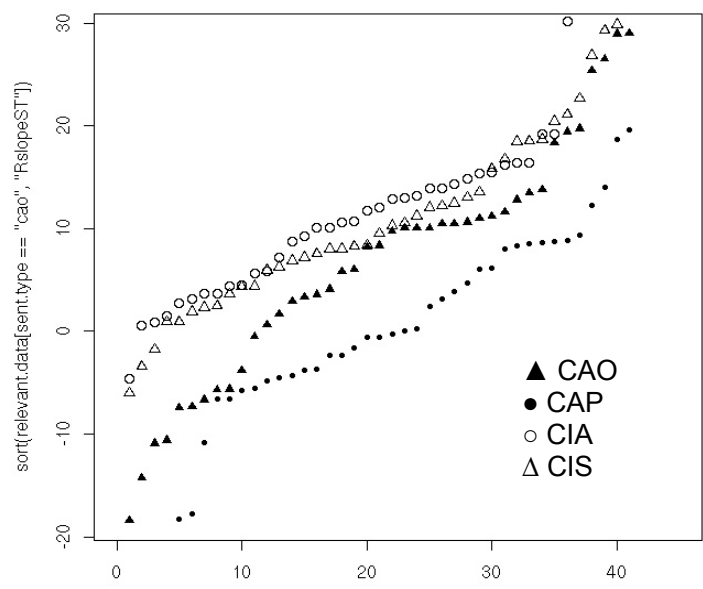

Linear Hypotheses

\section{Estim}

CAP-CAO $=0 \quad-7.9892$

CIA-CAO $=0 \quad 4.8406$

CIS-CAO $=0 \quad 3.9481$

CIA-CAP $=0 \quad 12.8298$

CIS-CAP $=0 \quad 11.9373$

$\mathrm{CIS}-\mathrm{CIA}=0$

$-0.8925$

Std. E

2.1006

$z$ value

1.796

1.488
4.756

2.6974

2.6570

4.493

$1 e-04=1 / 10000$
$\operatorname{Pr}(>|z|)$

0.000769 **

0.269306

0.438261

$<1 \mathrm{e}-04^{* * *}$

$<1 \mathrm{e}-04^{* * *}$

0.975893

Figure 3 : Rslope ST, contraste CAO-CAP-CIS-CIA : 160 observations

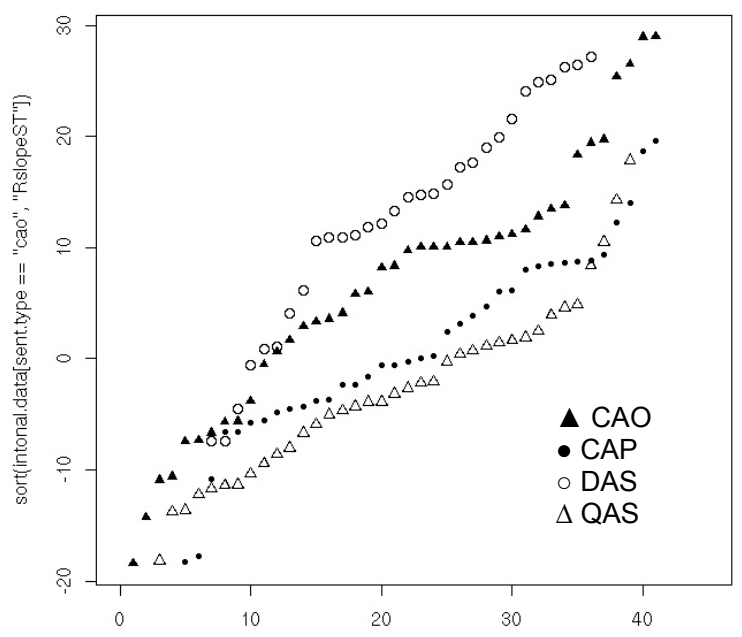

Linear Hypotheses

$\begin{array}{lllll} & \text { Estim. } & \text { Std. Er. } & \text { z value } & \operatorname{Pr}(>|\mathbf{z}|) \\ \text { CAP-CAO }=0 & -7.8688 & 2.9639 & -2.655 & 0.0399 \text { * } \\ \text { DAS-CAO }=0 & -8.3980 & 2.9639 & -2.833 & 0.0240 \text { * } \\ \text { QAS-CAO }=0 & 0.5316 & 3.0429 & 0.175 & 0.9981 \\ \text { DAS-CAP }=0 & -0.5293 & 2.9639 & -0.179 & 0.9980 \\ \text { QAS-CAP }=0 & 8.4004 & 3.0429 & 2.761 & 0.0296 \text { * } \\ \text { QAS-DAS }=0 & 8.9296 & 3.0429 & 2.935 & 0.0170 \text { * }\end{array}$




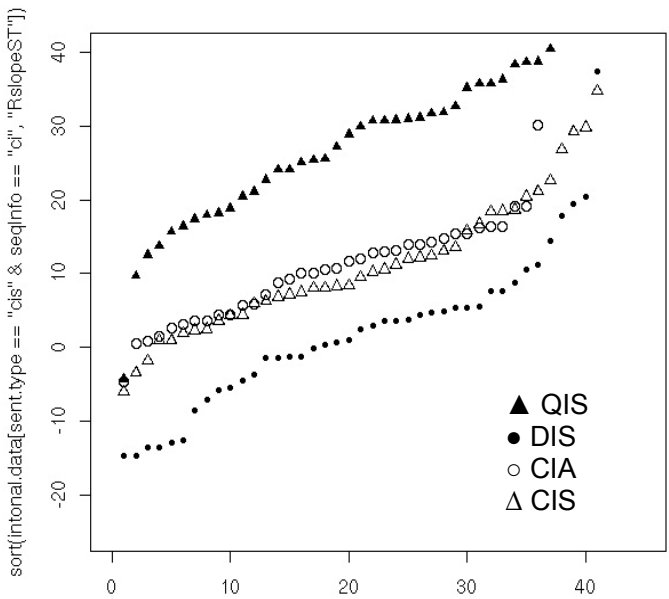

Linear Hypotheses

$\begin{array}{lllll} & \text { Estim. } & \text { Std. Er. } & \mathbf{z} \text { value } & \operatorname{Pr}(>|\mathbf{z}|) \\ \text { CIA-CIS=0 } & 0.8841 & 2.0505 & 0.431 & 0.972430 \\ \text { DIS-CIS=0 } & -8.8635 & 2.5010 & -3.544 & 0.002179^{* *} \\ \text { QIS-CIS=0 } & 17.1813 & 2.5107 & 6.843 & <1 \mathrm{e}-04^{* * *} \\ \text { DIS-CIA=0 } & -9.7476 & 2.5394 & -3.839 & 0.000687^{* * \star} \\ \text { QIS-CIA=0 } & 16.2972 & 2.5489 & 6.394 & <1 \mathrm{e}-04^{* * *} \\ \text { QIS-DIS=0 } & 26.0448 & 2.0053 & 12.988 & <1 \mathrm{e}-04^{* * *}\end{array}$

Figure 5. Rslope ST, contraste CIS-CIA-QIS-QIA : 159 observations

\section{Discussion}

L'exploitation des relevés de données et de certains des résultats ci-dessus permet d'apporter quelques éléments de réponse aux questions soulevées en 1.3.

\subsection{Nos configurations face aux propriétés des continuations mineures et majeures}

La première interrogation consistait à savoir si les patrons observés dans les configurations CIS-CIA pouvaient être montants et descendants et si leurs hauteurs maximales étaient systématiquement et significativement inférieures à celles observées dans les configurations CAO-CAP.

D'après le graphique de la figure 3, la très grande majorité des configurations CIS-CIA présentent une pente montante, soit 74 observations sur 78. Concrètement, cela est visible dans le fait que les points représentant les observations du corpus (ronds et triangles blancs) se situent au dessus de 0 sur la barre des ordonnées (valeurs de RslopeST $>0$ ) ${ }^{\text {vii }}$.

Nous avons poussé l'analyse un peu plus loin, pour essayer de déterminer si, dans notre corpus, il y avait une différence significative entre les configurations CIS-CIA $(\approx$ continuation mineure) et les configurations CAO-CAP ( $\approx$ continuation majeure), comme cela est censé être le cas si l'on suit la plupart des travaux distinguant les deux en disant que les continuations majeures montent plus haut que les continuations mineures.

Dans les données d'ensemble ${ }^{\text {viii }}$, nous avons observé les phénomènes suivants :

- La distribution des observations (montant/descendant) est assez différente pour les deux cas : 74 cas sur 78 de CIS-CIA ont un profil montant, mais seulement 49 cas sur 82 de CAO-CAP.

- Pour les cas où le profil est montant (valeur de Rslope ST $>0$ ), nous avons observé qu'il n'y avait pas un écart très important entre la moyenne de la pente pour les 74 observations de configuration CIS-CIA concernées et la moyenne de la pente pour les 49 observations de configuration CAO-CAP concernées (respect. 11,9 et 10,4). La comparaison des moyennes n'est pas suffisante pour savoir si la différence observée est significative.

- Toujours pour les cas où les profils sont montants, nous avons ensuite regardé les valeurs maximales en semi-tons sur les segments-cibles (maxST) pour chaque configuration (CIS-CIA vs CAO-CAP) et fait un test de Wilcoxon ${ }^{i x}$ pour estimer la significativité de la différence de leurs distributions. Il s'avère que la différence est jugée significative. Pour simplifier, dans nos 
données et selon ce test, les configurations CIS-CIA montantes montent significativement plus haut que les configurations CAO-CAP montantes.

Nous nous étions aussi demandé si les patrons observés dans les configurations CAO et CAP étaient toujours montants, caractéristique souvent présentée comme définitoire des contours de continuation majeure. Ce n'est pas le cas ici, puisque, comme nous l'avons remarqué, seulement 49 observations sur 82 présentent des pentes montantes (valeurs de RslopeST $>0$ dans les graphiques des figures 3 et 4 pour les ronds et les triangles noirs). Cette différence semble correspondre en partie aux deux (sous-)configurations : la configuration CAO est majoritairement montante et la configuration CAP majoritairement descendante. Les tableaux de contraste montrent très nettement que la différence est jugée significative (présence d'astérisques). En soi, le fait que ces résultats ne soient pas concordants avec l'analyse de Delattre n'est pas forcément une nouveauté, puisque des continuations qui pourraient être dites majeures ont été décrites comme descendantes dans d'autres travaux (cf. note 4). Ce qui appelle plus de réflexion, à notre avis, est la distribution de ces résultats par rapport à la construction du protocole : les patrons montants concernent préférentiellement les cas où la relation de discours entre les deux propositions est marquée par une conjonction et les patrons descendants les cas où elle ne l'est pas (asyndète).

Enfin, pour approfondir la comparaison entre nos configurations et les contours des continuations mineures et majeures, nous nous étions demandé (i) si les patrons produits par les sujets pour les configurations CIS-CIA étaient significativement différents des contours de question (QIS) et des contours conclusifs (DIS) correspondants et (ii) si les patrons produits par les sujets pour les configurations CAO-CAP étaient significativement différents des contours de question (QAS) et des contours déclaratifs conclusifs (DAS) correspondants, sachant que, dans la littérature, les patrons de question sont a priori montants et les patrons déclaratifs conclusifs descendants. Un certain nombre d'observations peuvent être faites à partir des figures 4 et 5 .

- Les configurations CIS et CIA, majoritairement montantes, ne présentent pas de différence notable entre elles au niveau de leur pente (les contrastes ne sont pas jugés significatifs dans les tableaux des figures 3 et 5). En revanche, elles sont significativement différentes des configurations DIS, majoritairement descendantes, et des configurations QIS qui présentent des valeurs de pente systématiquement supérieures (cf. graphique de la figure 5).

- Les configurations $\mathrm{CAO}$ et CAP ont été jugées significativement différentes entre elles du point de vue de leur pente (cf. figures 3 et 4). En termes de nombre d'observations, les configurations CAO sont plus souvent montantes (30 cas sur 41$)$ que les configurations CAP (19 cas sur 41). Les valeurs des pentes des configurations $\mathrm{CAO}$ sont systématiquement supérieures à celles des configurations CAP. De plus, les pentes des configurations DAS, bien qu'inférieures aux pentes des configurations CAP, ne sont pas jugées significativement différentes. De même, les pentes des configurations QAS qui sont supérieures aux pentes des configurations CAO.

\subsection{Marquage de la relation de discours et patron prosodique}

Ce second volet de l'étude est en fait un approfondissement de la remarque précédente concernant la différence observée entre les configurations CAO et les configurations CAP. Cette différence n'était pas attendue, et encore moins dans ce sens. En effet, les recherches préalables en français spontané sur les structures asyndétiques (section 1.1) et les exemples de continuations majeures trouvés dans la littérature (souvent des phrases complexes avec une conjonction), laissaient présager que, dans la majorité des cas, les sujets termineraient la première proposition avec une montée mélodique. En simplifiant, on pourrait résumer la situation ainsi : les patrons finaux des premières propositions dans des phrases complexes ne sont pas significativement différents des contours de question quand la relation de discours est marquée, et ils ne sont pas significativement différents des contours déclaratifs conclusifs quand la relation de discours est implicite.

Si l'expérience ne permet pas de tirer des conclusions définitives, elle oblige à s'interroger plus avant sur deux points : l'influence du marquage de la relation de discours entre deux propositions sur le 
patron prosodique final de la première proposition ; la spécificité prosodique ou non des phrases complexes par rapport notamment au contour de question et au contour déclaratif conclusif.

\section{$5 \quad$ Limites et perspectives}

Cette étude n'avait pas pour objectif d'apporter une réponse exhaustive aux questions soulevées, pour deux raisons principales.

D’une part, le protocole expérimental a obligé à faire un certain nombre de restrictions et de choix qu'il convient de dépasser dans une deuxième étape.

- Nous pensons notamment qu'il est nécessaire d'étudier un segment plus large que deux syllabes pour chercher, par exemple, si l'inversion de pente se fait au même niveau pour toutes les configurations contrastées, et si l'on peut, pour certaines configurations montantes, mettre en évidence un phénomène d'anticipation permettant de les différencier.

- La pente, telle que nous l'avons conçue (droite de régression) est un indicateur intéressant mais limité. Nous suggérons de poursuivre nos investigations en prenant en compte également les phénomènes de durée, pour voir si, par exemple, telle ou telle configuration se différencie systématiquement des autres par un allongement vocalique final. Les configurations CIS et CIA présentent-elles une finale significativement plus courte ou plus longue que les configurations $\mathrm{CAO}$ et CAP ? La configuration CAO présente-elle une finale significativement plus courte ou plus longue que la configuration QAS (l'analyse de la pente se suffit pas à les différencier) ?, etc.

- Nous avons laissé de côté la question de la concavité/convexité des courbes mélodiques, alors qu'il s'agit d'un critère important, chez Delattre, pour différencier notamment les continuations majeures et les questions. Cette propriété a été critiquée depuis, et jugée non pertinente (cf. Roméas 1992, Di Cristo 2010). Sur la base d'une étude plus précise des données de notre corpus qui irait plus loin qu'une analyse de la pente, nous pourrions y revenir éventuellement pour infirmer ou confirmer ces critiques. En effet, comme le montrent les exemples présentés dans les figures 2, la forme de la courbe mélodique est différente.

- Les résultats obtenus pour le contraste de la configuration CAO et de la configuration CAP ne concordent ni avec les premières définitions des continuations majeures, ni avec nos attentes, puisque nous pensions trouver en très grande majorité des patrons montants. Avant de généraliser, il convient de compléter l'expérience : il se trouve que, dans presque tous les cas, si l'on prend en compte le contexte proposé au sujet, les phrases complexes fabriquées présentent la première proposition comme étant rhématique (portant l'information de premier plan). Les résultats obtenus ne valent donc que pour des configurations $\mathrm{CAO}$ et CAP rhématiques. Il s'agit désormais de savoir si la distribution observée se vérifie aussi quand c'est la deuxième proposition qui constitue le rhème.

- Enfin, toujours à propos de la différence observée entre la configuration CAO et la configuration CAP. L'expérience n'a pris en compte que des cas dans lesquels la relation de discours en jeu était une relation causale. De plus, pour les phrases de type $\mathrm{CAO}$, la dépendance syntaxique était à chaque fois une coordination. Pour une étude plus systématique du phénomène, il faudrait compléter l'expérience en proposant aux sujets des phrases complexes mettant en jeu un choix plus important de relations de discours, ainsi qu'une alternance systématique au niveau du type de dépendance syntaxique entre les deux propositions (coordination, subordination, juxtaposition).

D'autre part, cette étude s'insère dans un projet plus général, le projet INTONALE, portant sur la différence de production des contours de continuation en L1 (le français) et en L2 (l'anglais). Les études comparant les particularités prosodiques de l'anglais et du français mettent en évidence, de manière plus ou moins détaillée, un certain nombre de tendances et de différences entre les deux langues (voir par 
exemple Vaissière 2002 : 10 sq., Delattre $1961: 62,1966: 10$ ). Dans une étude consacrée à la partie «anglais » de notre corpus (Bartkova, Bonneau, Colotte \& Dargnat, à par. 2012), nous avons pu remarquer que les contrastes entre types de configurations n'étaient pas tout à fait les mêmes, dans le cas des locuteurs français parlant anglais (mêmes locuteurs que pour l'expérience en français) et des locuteurs anglais parlant anglais (locuteurs natifs originaires de Newcastle). Sans entrer dans le détail, à ce stade, on peut mentionner les deux observations suivantes :

i) dans les cas de l'anglais L2, les pentes observées pour les configurations de type CIS-CIA (SN sujets) ne sont pas significativement différentes des pentes observées pour les configurations de type CAP-CAO (fin de proposition 1 dans une phrase complexe) ;

ii) à l'inverse de ce qui s'est passé pour le français L1, l'anglais L2 ne montre pas de différence significative en ce qui concerne les pentes des configurations de type CAO (avec coordonnant) et les pentes des configurations de type CAP (parataxes). Les locuteurs français paraissent moins influencés en situation de L2 qu'en situation de L1 par la présence d'une conjonction de coordination.

Cela appelle bien entendu une étude plus approfondie, tant du point de vue qualitatif que quantitatif, et une comparaison systématique avec le groupe de contrôle que constituent les enregistrements effectués auprès de locuteurs anglais natifs, pour voir si la prononciation des apprenants en ce qui concerne les configurations étudiées présente un système de contours particulier ou si, plus simplement, elle emprunte au français L1, langue d'origine, et à l'anglais L1, langue cible des apprenants.

\section{Références bibliographiques}

Avanzi, M. (2011). L'interface prosodie/syntaxe en français. Dislocations, incises et asyndètes. Thèse des universités de Neuchâtel et de Paris 10. non publiée.

Avanzi, M. \& Lacheret-Dujour, A. (2010). Micro-syntaxe, macro-syntaxe : une prosodie toujours transparente ? L'exemple des périodes asyndétiques en français parlé. In Béguelin, M.-J., Avanzi, M., Corminboeuf, G. (éds), La parataxe, vol.2 : Structures, marquages et exploitation discursive. Bern : Peter Lang, 339-364.

Autesserre, D., Di Cristo, A. (1972). Recherches psycho-sémantiques sur l'intonation de la phrase française. Travaux de l'Institut de Phonétique d'Aix, 1, 61-98.

Bartkova, K., Bonneau, A., Colotte, V., Dargnat, M. (à par. 2012). Productions of 'Continuation contours' by French speakers in L1 (French) and L2 (English). Speech Prosody 2012, Special Session 'Prosody and languages in contact', 22-25 May 2012, Shangaï, 4 p.

Benzitoun, C. (2010). Comment tirer profit de la para-taxe ? In Béguelin, M.-J., Avanzi, M., Corminboeuf, G. (dir.), La parataxe. Entre dépendance et intégration, vol. 1. Bern : Peter Lang, 153-174.

Beyssade, C., Delais-Roussarie, E., Doetjes, J., Marandin, J.-M., Rialland, A. (2006). Prosody and Information in French. In Corblin, F., de Swart, H. (eds), Handbook of French Semantics. Stanford : CSLI, 477-499.

Choi-Jonin, I., Delais-Roussarie, E. (2006). L'association de propositions sans marque segmentale en français parlé : étude syntactico-sémantique et prosodique. Faits de langue, 28, 83-94.

Chen, A. (2007). Language-specificity in the perception of continuation intonation. In Gussenhoven, C., Riad, T. (eds), Tones and Tunes II. Berlin : Gruyter, 107-142.

Culicover, P. \& Jackendoff, R. (1997). Semantic Subordination despite Syntactic Coordination. Linguistic Inquiry, 28/2, 195-217.

Corpus Recorder (logiciel utilisé pour l'enregistrement du corpus Intonale), développé au sein de l'équipe «Parole » du Loria à Nancy : http://raweb.inria.fr/rapportsactivite/RA2011/parole/uid63.html

Dargnat, M. (2008). Constructionnalité des parataxes conditionnelles. In Durand J., Habert B., Laks B. (éd.), actes du Congrès Mondial de Linguistique Française (CMLF 08), Paris Institut de Linguistique Française, 2467-2482.

Dargnat, M., Bonneau, A., Colotte, V. et Bartkova, K. (2011). Intra and Inter clausal Non-conclusive Slopes in French: First Results. Poster présenté à Experimental and Theoritical in Prosody (ETAP 2) 'Prosody in Context', 23-25 septembre 2011, Montréal. 
Dargnat, M., Bartkova, K., Bonneau, A. et Colotte, V. (2011). Non-conclusive Slopes in French: First Results. Poster présenté à Prosody and Discourse Interface (IDP 2011), 12-14 septembre 2011, Manchester.

Dargnat, M. \& Jayez, J. (2010). La cohésion parataxique : une approche constructionnelle. In Béguelin, M.-J., Avanzi, M., Corminboeuf, G. (éd.), La Parataxe, Structures, marquages et explications discursives, Berne, Peter Lang, tome 2, 61-93.

Delais-Roussarie, E. (2005). Phonologie et grammaire : études et modélisation des interfaces prosodiques. Thèse d'Habilitation à Diriger des Recherches de l'Université de Toulouse 2.

Delais-Roussarie, E. (2011). Les contours montants non terminaux du français. Contraste, opposition, distribution. Support de conférence : http://mathilde.dargnat.free.fr/INTONALE/intonale-web.html, séminaire SémantiqueMorphologie-Syntaxe, Université Nancy 2 \& ATILF, 15 avril.

Delattre, P. (1961). La leçon d'intonation de Simone de Beauvoir, étude d'intonation déclarative comparée. The French Review, 35/1, 59-67.

Delattre, P. (1966). Les dix intonations de base du français. The French Review, 40/1, 1-14.

Deulofeu, H. J. (1977). La syntaxe et les constructions binaires. Recherches sur le français parlé, 1, 30-61.

Deulofeu, H. J. (1989). Les couplages de constructions verbales en français parlé : effet de cohésion discursive ou syntaxe de l'énoncé ? Recherches sur le Français Parlé, 9, 111-141.

Deulofeu, H. J. (1999). Recherches sur les formes de la prédication dans les énoncés assertifs en français contemporain (le cas des énoncés introduits par le morphème que). Thèse d'Etat de l'Université Paris 3.

Di Cristo, A. (1998). Intonation in French. In Hirst, D., Di Cristo, A. (eds), Intonation Systems: A survey of Twenty Languages. Cambridge : Cambridge University Press, 195-218.

Di Cristo, A. (2004). La prosodie au carrefour de la phonétique, de la phonologie et de l'articulation formesfonctions. Travaux interdisciplinaires du Laboratoire Parole et Langage, 23, 67-211.

Di Cristo, A. (2010). A propos des intonations de base du français. Manuscrit.

Giron, S. (2004). Inventaire et classement des constructions verbales dans un corpus de français parlé dans l'Allier. Thèse de doctorat de l'Université Clermont-Ferrand II. (Un important corpus (le corpus Allier) a été recueilli et transcrit selon les normes du GARS).

Glikman, J. (2009). Parataxe et Subordination en Ancien Français: Système syntaxique, variantes et variation. Thèse de doctorat de l'Université Paris X et de l'Université de Potsdam.

Jayez, J., Dargnat, M. (à par. 2012). The Semantics of French Continuative Rises in SDRT. In Benz, A., Stede , M. (eds), actes du Workshop Constraints in Discourse. Amsterdam/Philadephia : Benjamins. 20 p.

Jun, S.-A. \& Fougeron, C. (2000). A Phonological model of French intonation. In Botinis, A. (ed.), Intonation: Analysis, Modeling and Technology. Dordrecht : Kluwer Academic Publishers, 209-242.

INTONALE, (Production, Perception et apprentissage des contours prosodiques en L1 et L2), projet 2009-2011, financé par le Le Comité de Coordination et d'Orientation Scientifique Lorrain (CCOSL), qui rassemble les établissements universitaires et les organismes de recherche lorrains (Universités, CNRS, INRA, INRIA, INSERM) ainsi que le CHU (Centre Hopitalier Universitaire). Participants : ATILF : K. Bartkova, M. Dargnat, LORIA : A. Bonneau, V. Colotte. Page provisoire : $\underline{\text { http://mathilde.dargnat.free.fr/INTONALE/intonale-web.html }}$

Lacheret, A. (2007). Prosodie-discours : une interface à multiples facettes. Nouveaux Cahiers de linguistique française, 28, 7-40.

Marandin, J.-M. (2006). Contours as Constructions. In Schoenefeld, D. (éd.), Constructions all over : case studies and theoritical implications. http://elanguage.net/journals/index.php/constructions/article/view/25/30

Marandin, J.-M. (2010). Quelles expériences pour étudier le sens de la prosodie ? Support de conférence : http://mathilde.dargnat.free.fr/INTONALE/intonale-web.html, séminaire Sémantique-Morphologie-Syntaxe, Université Nancy 2 \& ATILF, 26 mars.

Martin, P. (1987). Prosodic and rythmic structures in French. Linguistics, 25, 925-949.

Martin, P. (2009). L'intonation du français. Paris : Armand Colin. 
Mertens, P. (1987). L'intonation du français. De la description linguistique à la reconnaissance automatique. Thèse de l'Université de Louvain.

Mertens, P. (1990). Intonation. In Blanche-Benveniste, C. et al. Le français parlé, études grammaticales. Paris : CNRS éditions, 159-176.

Portes, C., Bertrand, R., Espesser, R. (2007). Contribution to a grammar of intonation in French, Form and function of three rising patterns. Nouveaux Cahiers de Linguistique Française, 28, 155-162.

Post, B. (2000). Tonal and Phrasal Structures in French Intonation. Thèse de l'université de Nimègue.

Praat. (2011). Doing phonetics by computer (Boersma, P., Weenink, D.), version 5.3.02.

http://www.fon.hum.uva.nl/praat

R. (logiciel d'analyse statistique) The R Project for Statistical Computing, http://www.r-project.org/ .

Riegel, M., Pellat, J.-C., Rioul, R. (2009). Grammaire méthodique du français. Paris : Presses Universitaires de France.

Roméas, P. (1992). L'organisation prosodique des énoncés en situation de dialogue homme-machine. Thèse de l’Université de Provence.

Rossi, M. (1999). L'intonation : le système du français. Paris : Ophrys.

Strip Tease, DVD des émissions de Marco Lamensch et Jean Libon, 2004 pour les volumes 1,2, 3 /MK2 SA et Anne Carter et 2006 pour les volumes 4,5,6 / MK2 SA et Aconit Productions.

Vaissière, J. (2002). Cross-linguistic prosodic transcription: French vs. English. In: Volskaya, N. B., Svetozarova, N. D., Skrelin, P. A. (éd.), Problems and methods of experimental phonetics. In honour of the 70th anniversary of Pr. L.V. Bondarko. Saint-Pétersbourg : Saint-Pétersburg State University Press, 147-164.

WinSnoori (2009). Free Software for Speech Analysis (Yves Laprie), version 1.34.07. http://www.loria.fr/ laprie/WinSnoori 
i Cette démarche s'inscrit dans un projet plus large (projet INTONALE) visant à étudier systématiquement et expérimentalement deux schémas de continuation en français (L1) et en anglais (L1 et L2). L'analyse contrastive des deux langues est présentée ailleurs de manière plus détaillée (Bartkova, Bonneau, Colotte \& Dargnat, à par. 2012). Dans les perspectives évoquées dans la conclusion, nous rapportons quelques observations sur ce point.

ii Les termes continuation simple et continuation groupante sont ceux proposés par Delattre en 1961. Il les remplacera ensuite respectivement par continuation mineure et continuation majeure. Cette distinction est parfois critiquée ou jugée insuffisante (Di Cristo 2010, Vaissière $2002: 8$ ), mais elle reste tout de même une référence pour aborder le problème. Delais-Roussarie (2011) fait remarquer que même si les approches phonologiques, comme celles de P. Mertens, P. Martin ou B. Post, ne proposent pas cette distinction dans leur inventaire de patrons, elle découle des «principes de dominance chez Mertens, de la distribution chez Post et de la distribution et de la structure chez Martin ».

iii Delattre fait une remarque très intéressante sur ce point dans son article de 1961. «Souvent, écrit-il, dans l'absolu, un B [=une continuation majeure] n'atteint pas plus haut qu'un A [=une continuation majeure] assez proche. Il faut donc que la perception du contraste soit associée à un autre facteur : la pénultième est alors plus basse, relativement à la finale, dans B que dans A. » (62). Ici, nous n'avons pas étudié le phénomène, mais le protocole a été construit de sorte que cela soit possible. Pour une discussion récente sur la différence des hauteurs maximales pour les deux continuations, se reporter à Di Cristo (2010:5-6).

iv La littérature a effectivement tendance à définir la continuation majeure du point de vue de sa forme, essentiellement montante. Mais, dans le détail, les choses sont moins catégoriques. On trouve par exemple des « majeures descendantes » chez Delais-Roussarie (2011: 21-22) et Di Cristo (2010: 17). Voir également les propos de Delattre (1961 : 60 sq.).

v Packadge lme4.

vi Fonction glht du packadge multcomp.

vii Un relecteur s'interroge sur la possibilité d'un contour plat, c'est-à-dire d'un « effacement » de la montée de continuation, en particulier pour les cas associés à de la continuation mineure. Dans notre corpus, il y en a peu d'exemples de ce type. Cela reste à analyser pour les corpus de français spontané.

viii Le tableau de l'ensemble des données recueillies automatiquement n'est pas représenté ici pour des raisons de place. Dans ce tableau, nous avons les relevés de F0 toutes les $10 \mathrm{~ms}$ pour tous les enregistrements, ainsi qu'un détail de la valeur minimale (minST) et de la valeur maximale (maxST) en semi-tons pour chaque segment analysé (en gras dans les exemples du tableau 1).

ix Le test de Wilcoxon, pour des échantillons non appariés, n'est pas sensible au fait que les deux échantillons n'ont pas la même taille (ici, 74 cas pour CIS-CIA et 49 cas pour CAO-CAP). 